\title{
Targeted Therapy in Biliary Tract Cancers
}

\section{Amartej Merla, MD \\ Kenneth G. Liu, MD \\ Lakshmi Rajdev, MD*}

\author{
Address \\ *Department of Medical Oncology, Montefiore Medical Center, 1695 Eastchester \\ Road, 2nd Floor, Bronx, NY, 10461, USA \\ Email: trajdev@montefiore.org \\ Published online: 13 August 2015 \\ (C) The Author(s) 2015. This article is published with open access at Springerlink.com
}

This article is part of the Topical Collection on Upper Gastrointestinal Cancers

Amartej Merla and Kenneth G. Liu contributed equally to this work.

Keywords Biliary tract cancer - Targeted therapy $\cdot$ EGFR $\cdot$ VEGF $\cdot$ Trial

\section{Opinion statement}

A paradigm shift towards molecular-based, personalized cancer therapeutics has occurred in recent years and a number of targeted drugs have emerged. Various targeted therapies like erlotinib, trastuzumab, and cetuximab have been approved in lung, breast, and colon cancers, respectively. Numerous clinical trials involving targeted drugs in biliary tract cancers are currently in progress, though none have been approved for this disease. Biliary tract cancers are divided into separate entities both anatomically and in terms of pathogenesis but are grouped together in most trials given their rarity. Combination chemotherapy involving cisplatin and gemcitabine is the current standard of care in the metastatic setting. In this review, we will discuss the various molecular pathways implicated in biliary tract cancers and potential therapeutic targets.

\section{Classification}

Biliary tract cancers (BTC) are a heterogeneous group of cancers that are clinically and genetically divergent. BTC can be divided anatomically into gallbladder cancers (GBC), intrahepatic cholangiocarcinomas (IHCC), hilar cholangiocarcinomas (HCC), and extrahepatic cholangiocarcinomas (EHCC) [1]. HCC, also called Klatskin's tumors as they were first described by Gerald Klatskin in 1965, are tumors that arise at the bifurcation of the common hepatic duct [2]. The American Joint
Commission on Cancer (AJCC) 7th edition classified them as different types with separate staging systems, though the systemic management options at present remain similar. Histologically, BTC are classified as adenocarcinoma (90\% of cases), papillary, mucinous, clear cell, signet-ring cell, adenosquamous, squamous, small cell, and undifferentiated carcinomas [3]. The exact molecular mechanisms leading to BTC remain unknown. 


\section{Epidemiology}

\section{Prognosis}

BTC are rare malignancies with poor prognosis, with GBC being the most common among all BTC [4]. Population-based data from the National Cancer Institute's Surveillance, Epidemiology, and End Results (SEER) program estimated 7865 newly diagnosed cases of GBC from 1992 to 2009 in the USA. Incidence is higher in females, and higher incidence rates are seen in blacks and Asians compared to whites [5]. Mortality rates are highest in Chile, India, and Eastern Europe, suggesting geographical and racial differences [6, 7]. For IHCC and EHCC, incidence rates in the USA are 0.88 per 100,000 and 0.72 per 100,000, respectively, based on data from the National Center for Health Statistics [8]. The incidence of IHCC worldwide is increasing, while that of EHCC appears to be decreasing $[9,10]$.

Risk factors for BTC include parasitic infections by liver flukes, gallstones, diabetes mellitus, obesity, alcohol, inflammatory bowel disease, bile duct cysts, smoking, and hepatitis B and C [11-15]. The presence of gallstones is by far the most important risk factor. The exact mechanism by which gallstones cause carcinogenesis is unknown but is probably due to persistent inflammation leading to dysplasia and accumulation of loss of heterozygosity at various tumor suppressor genes $[16,17]$. The presence of gallbladder adenoma has also been proposed as a risk factor [18].

GBC have the worst prognosis among all BTC. For resectable GBC, the median overall survival (OS) differs somewhat depending on the actual study. A population-based analysis using SEER data concluded that median OS for resectable GBC is only 16 months [19]. For IHCC, OS after resection is 33 months, and the 1-, 3-, and 5-year OS estimates are 82.3, 47.1, and $32.9 \%$, respectively. Another study reported OS of 18 months after R0 resection for node-positive disease $[20,21]$. Five to ten percent of patients were alive 5 years after being diagnosed with advanced IHCC [10]. For surgically resectable HCC, the median OS is 30 months in patients undergoing combined partial hepatic resection and 24 months in those having bile duct resection only [22]. In contrast, despite an increase in treatment options, locally advanced or metastatic BTC have median OS approaching only 1 year, illustrating the need for better therapy [23].

\section{Chemotherapy}

Treatment of early BTC is surgery, which offers potential cure. In locally advanced HCC, neoadjuvant chemoradiation followed by liver transplantation across 12 centers in the USA was shown to improve 5-year recurrence-free survival to $65 \%$ [24]. Adjuvant therapy remains controversial and no regimen has yet been accepted as a standard. Only about $10 \%$ of BTC are operable at the time of diagnosis. For advanced, inoperable BTC, systemic therapy is the only option. Poor prognostic factors after resection 


\section{Targeted therapy}

include the presence of lymph node metastases, positive margins, and poor differentiation $[25,26]$.

The use of chemotherapy over best supportive care was first supported in a clinical trial comparing combination of 5-fluorouracil and etoposide vs. best supportive care. The median OS in the chemotherapy group was 6 months, compared to only 2.5 months in the supportive care group [27].

The current standard of care in the metastatic setting is based on the ABC-01 and $\mathrm{ABC}-02$ trials from the UK $[23,28]$. ABC-01, a phase II clinical trial, demonstrated improvement in 6-month progression-free survival (PFS) favoring cisplatin and gemcitabine compared to gemcitabine alone ( $8.0 \mathrm{vs.}$ 4.0 months) [28]. ABC-02, a phase III trial, showed an improvement in OS, with combination therapy of cisplatin and gemcitabine (11.7 months) compared to gemcitabine alone (8.1 months) (hazard ratio (HR) 0.64, $95 \%$ confidence interval (CI) 0.52-0.80; log rank $p<0.001$ ) [23]. The Japanese BT-22 trial produced results similar to the ABC- 02 trial, in which 84 patients were randomized to either gemcitabine alone or to the combination of cisplatin and gemcitabine [29]. A subsequent meta-analysis concluded that BTC patients treated with gemcitabine combined with platinum agents (cisplatin or oxaliplatin) have better outcomes compared to those not treated with this regimen [30].

Recent studies revealed multiple clinically targetable mutations in BTC. Many molecular pathways are implicated in carcinogenesis, and agents targeting these pathways have shown some efficacy in BTC cell lines [31, 32]. In a recent presentation on comprehensive genomic profiling of biliary tract cancers by Ross et al. at the 2015 Gastrointestinal Symposium, multiple genomic alterations and tumor-specific differences were noted. Next-generation sequencing was performed on 554 BTC specimens. IHCC, EHCC, and GBC share genomic alterations in cell cycle regulation (CDKN2B) and chromatin remodeling (ARID1A). IHCC harbor FGFR fusions, IDH1/2 substitutions, BRAF substitutions, and MET amplification with a low KRAS mutation frequency. EHCC and GBC both have ERBB2 amplifications and PIK3CA/mTOR pathway alterations, but KRAS mutation frequencies are high in EHCC and low in GBC [33•]. Whole exome and targeted gene sequencing in earlier studies of GBC also revealed frequent mutations in TP53, KRAS, and ERBB3 [34-36]. Microsatellite instability (MSI), which is well known in the pathogenesis of colon cancer, does not appear to play a role in BTC [37]. Various targeted agents for BTC have been tested in several phase I and II clinical trials (Table 1).

\section{ErbB family signaling pathway}

There are four distinct receptors that belong to the ErbB family of receptor tyrosine kinases: epidermal growth factor receptor (EGFR, also known as ErbB1/HER1), ErbB-2 (neu, HER2), ErbB-3 (HER3), and ErbB-4 (HER4). The intracellular tyrosine kinase domain of ErbB receptors is highly conserved, while the extracellular domains are less conserved among the four receptors, suggesting different specificities in ligand binding. ErbB receptors are important in the 


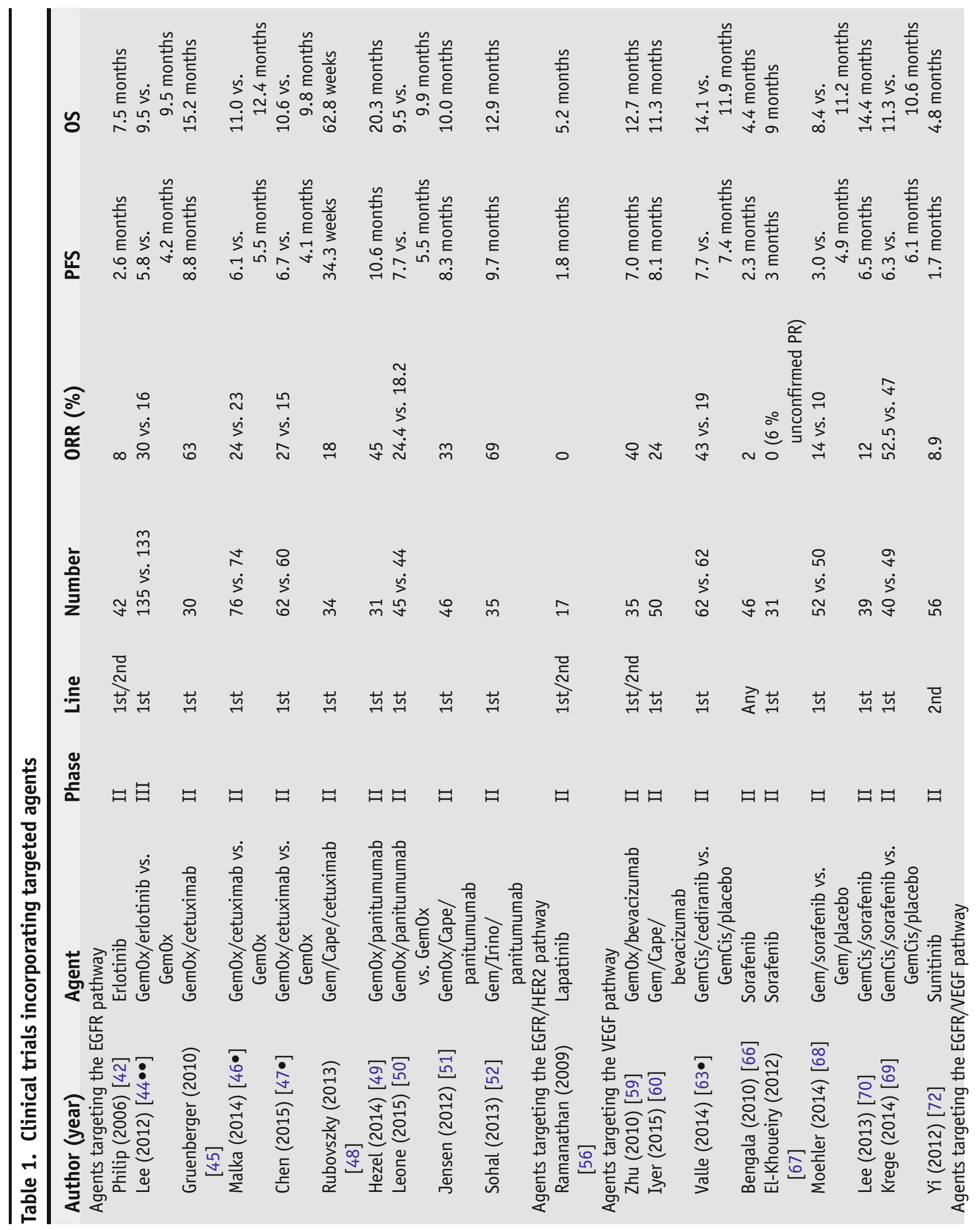




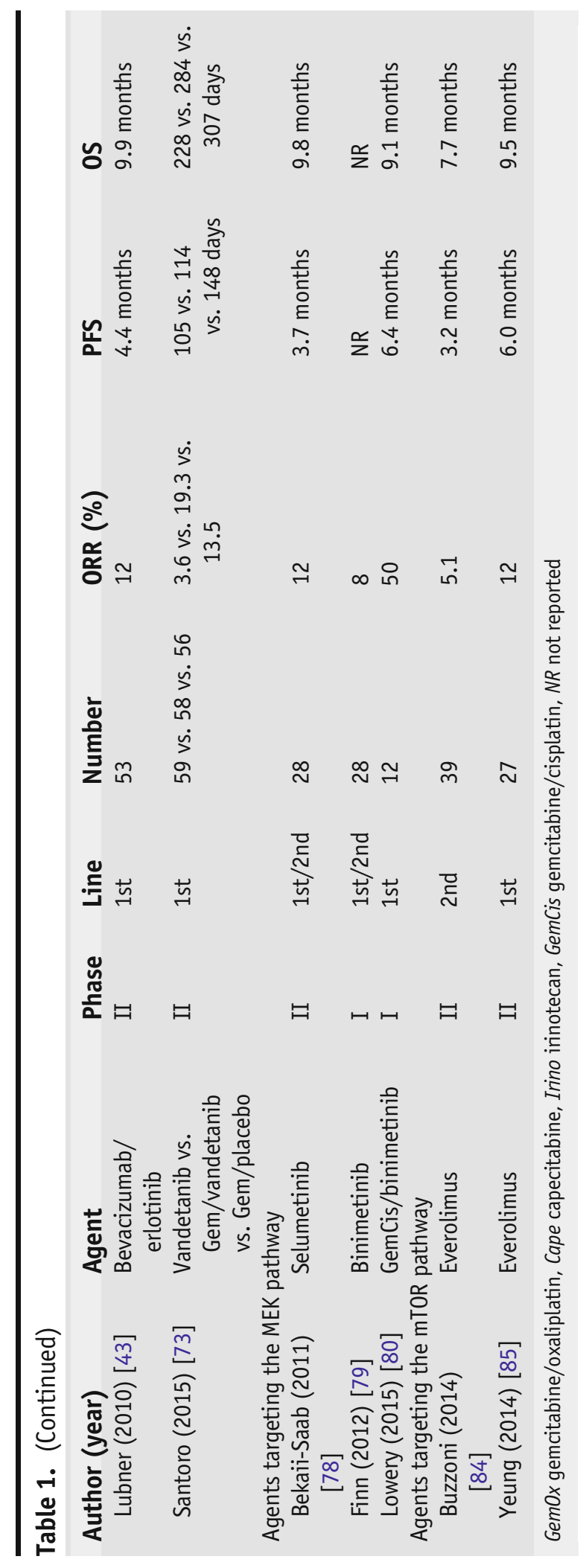


development of different organs, and the interaction of ErbB receptors and their ligands are associated with tumorigenesis [38]. EGFR and HER2 inhibitors tested in BTC cell lines in combination with gemcitabine have shown promising activity [31], and they are being investigated in clinical trials, either alone or in combination with chemotherapy (Table 2).

\section{EGFR pathway}

EGFR is commonly expressed in BTC and is found in $100 \%$ of IHCC, $52.6 \%$ of EHCC, and $38.5 \%$ of GBC in one study [31]. A Japanese cohort study demonstrated that EGFR expression in IHCC is significantly associated with poor prognosis, while its presence in EHCC may represent tumor progression and invasion [39]. Because EGFR antibodies and inhibitors have been proven clinically to be efficacious for many cancers, they have also been investigated in BTC.

\section{Erlotinib}

Erlotinib is an orally active, potent, selective, and reversible inhibitor of EGFR. Its use in various cancers, such as lung, head, and neck cancers, are well documented. EGFR overexpression is seen in several BTC studies and has been studied as a therapeutic target [40, 41]. Several phase II trials have investigated the role of erlotinib in the management of advanced BTC. Philip et al. evaluated 42 patients with advanced BTC and showed median PFS of 2.6 months and median OS of 7.5 months. However, more

\begin{tabular}{|c|c|c|c|c|}
\hline Drug & Class & Phase & Arms & NCT number \\
\hline Cetuximab & EGFR antibody & II & Gemcitabine + oxaliplatin with or without cetuximab & NCT01267344 \\
\hline Afatinib & EGFR inhibitor & I & Cisplatin + gemcitabine + afatinib (single arm) & NCT01679405 \\
\hline Cediranib & VEGF inhibitor & II & FOLFOX with cediranib (single arm) & NCT01229111 \\
\hline Regorafenib & Multikinase inhibitor & II & Regorafenib (single arm) & NCT02053376 \\
\hline Regorafenib & Multikinase inhibitor & I/II & Gemcitabine + oxaliplatin + regorafenib (single arm) & NCT02386397 \\
\hline Regorafenib & Multikinase inhibitor & II & Regorafenib (single arm) & NCT02053376 \\
\hline Regorafenib & Multikinase inhibitor & II & Regorafenib (single arm) & NCT02115542 \\
\hline Ponatinib & Multikinase inhibitor & II & Ponatinib (single arm) & NCT02265341 \\
\hline Pazopanib & Multikinase inhibitor & II & Pazopanib (single arm) & NCT01855724 \\
\hline Pazopanib & Multikinase inhibitor & II & Pazopanib + gemcitabine (single arm) & NCT01855724 \\
\hline Trametinib & MEK inhibitor & II & Trametinib vs. 5-FU & NCT02042443 \\
\hline Trametinib & MEK inhibitor & II & Trametinib (single arm) & NCT01943864 \\
\hline Selumitinib & MEK inhibitor & II & Cisplatin + gemcitabine with or without selumitinib & NCT02151084 \\
\hline Binimetinib & MEK inhibitor & I/II & Cisplatin + gemcitabine + binimetinib (single arm) & NCT01828034 \\
\hline Vismodegib & Hh pathway inhibitor & I & Vismodegib (single arm) & NCT00968981 \\
\hline R04929097 & Notch inhibitor & I & R04929097 (single arm) & NCT01096355 \\
\hline BGJ 398 & FGFR inhibitor & II & BGJ 398 (single arm) & NCT02150967 \\
\hline Ponatinib & FGFR inhibitor & II & Ponatinib (single arm) & NCT02265341 \\
\hline AG-881 & IDH inhibitor & $\mathrm{I}$ & AG 881 (single arm) & NCT02481154 \\
\hline$A G-120$ & IDH inhibitor & I & AG-120 (single arm) & NCT02073994 \\
\hline
\end{tabular}


than $50 \%$ of these patients received prior therapy; thus, these patients might have worse characteristics compared to average. In addition, 7 (17\%) patients were progression free at 24 weeks and all were EGFR/ HER1 positive, but the correlation between response and EGFR/HER1 status could not be measured accurately given the small sample size [42]. Lubner et al. investigated erlotinib in combination with bevacizumab, a vascular endothelial growth factor (VEGF) inhibitor. Nine patients $(18 \%)$ achieved an at best response of partial response (PR), 6 (12\%) of which had responses confirmed 4 weeks after their initial responses were observed. Median OS was 9.9 months, similar to that of historical controls [43].

In an open-label, randomized phase III study, Lee et al. assessed the efficacy of the addition of erlotinib to gemcitabine and oxaliplatin as firstline therapy for metastatic BTC. Two hundred sixty-eight South Korean patients were assigned in a 1:1 ratio to receive gemcitabine and oxaliplatin with or without erlotinib. Median PFS was 5.8 months in the chemotherapy plus erlotinib group and 4.2 months in the chemotherapy alone group (HR 0.80, $95 \%$ CI 0.61-1.03, $p=0.087$ ). OS was the same (9.5 months) for both groups (HR 0.93, $95 \%$ CI 0.69-1.25, $p=0.611$ ). Subgroup analysis of PFS in cholangiocarcinoma (HR 0.73) and GBC/ampulla of Vater (HR 0.99) cancers showed that much of the improvement in PFS came in the cholangiocarcinoma subgroup. This trial is the first phase III trial to assess a targeted therapy plus chemotherapy combination for patients with advanced BTC. However, the addition of erlotinib did not produce a significant improvement in median PFS. The study was flawed as it was not adequately powered and had a small sample size. The study was also limited to patients from South Korea and might not be generalizable to the non-Asian population $[44 \bullet \bullet]$.

\section{Cetuximab}

Cetuximab, an anti-EGFR antibody, is evaluated in combination with gemcitabine/oxaliplatin chemotherapy in BTC in several phase II trials. An earlier study showed encouraging results in locally advanced or metastatic BTC patients who received gemcitabine/oxaliplatin plus cetuximab. Of the 30 enrolled patients, $10 \%$ patients achieved CR, while $53 \%$ achieved PR. Nine patients underwent potentially curative secondary resection after major response to therapy [45]. However, subsequent studies failed to demonstrate a similar benefit. Malka et al. recruited 150 patients with locally advanced or metastatic BTC, who were randomized to receive gemcitabine/oxaliplatin with or without cetuximab in the first-line setting. Median PFS was 6.1 months (95 \% CI 5.1-7.6) with cetuximab and 5.5 months (95 \% CI 3.7-6.6) without cetuximab, while median OS was 11.0 months (95\% CI 9.1-13.7) with cetuximab and 12.4 months (95\% CI 8.6-16.0) without cetuximab. Despite being well tolerated, the addition of cetuximab to gemcitabine and oxaliplatin did not improve outcomes when used as first-line treatment for patients with advanced BTC [46•]. Chen et al. investigated whether KRAS mutation status influences response to cetuximab. One hundred twenty-two patients were randomized to receive gemcitabine/oxaliplatin with or without cetuximab. Median PFS was 6.7 months with cetuximab and 4.1 months without 
cetuximab ( $p=0.05)$, while median OS was 10.6 months with cetuximab and 9.8 months without cetuximab ( $p=0.91$ ). KRAS mutations, detected in $36 \%$ of tumors, did not affect the objective response rate (ORR) or PFS [47•].

Cetuximab was also evaluated in combination with gemcitabine and capecitabine in the first-line setting, but ORR was only $17.6 \%$, with a median PFS of 32.3 weeks and OS of 62.8 weeks [48].

\section{Panitumumab}

Panitumumab is a fully humanized anti-EGFR antibody. Given its role in KRAS wild-type colorectal cancers, it has also been studied in the first-line setting in advanced BTC phase II trials. Hezel et al. evaluated gemcitabine and oxaliplatin in combination with panitumumab in KRAS wild-type metastatic BTC. ORR was $45 \%$, median PFS was 10.6 months, and median OS was 20.3 months [49]. An Italian group recently presented preliminary data in a phase II randomized, open-label trial, in which 89 patients with KRAS wild-type metastatic or unresectable BTC were randomized to receive gemcitabine and oxaliplatin with or without panitumumab. No statistical significance for median PFS and OS was noted between the two arms, though there was a trend toward improved ORR and PFS in the arm with panitumumab [50]. Jensen et al. looked at the regimen gemcitabine, oxaliplatin, and capecitabine in combination with panitumumab in KRAS wild-type advanced BTC. ORR was $33 \%$ and the disease control rate was $86 \%$. Median PFS was 8.3 months and median OS was 10.0 months. The treatment was overall tolerable with some EGFR-related skin adverse events [51]. Sohal et al. reported data involving yet a different regimen, gemcitabine and irinotecan in combination with panitumumab. A promising ORR of $69 \%$ was noted, though median PFS of 9.7 months and OS of 12.9 months were relatively similar to previous trials [52]. However, given that these phase II trials had small sample sizes, the role of panitumumab in biliary tract cancers remains to be defined.

\section{HER2 pathway}

HER2 belongs to the ErbB family of receptor tyrosine kinases. It is overexpressed in about $10 \%$ of GBC and $26.3 \%$ EHCC in one study [31]. HER2 was first studied in breast carcinoma, and its overexpression in human breast carcinomas is associated with a more aggressive course of disease [53]. In an experimental tumor model, overexpression of HER2 in the basal layer of the biliary tract epithelium resulted in GBC in $100 \%$ of transgenic mice by 3 months of age and in other BTC at a $30 \%$ incidence rate [54]. Agents targeting HER2 are being tested in BTC patients given its efficacy in other cancers, though none clinically proven to improve outcomes thus far.

\section{Trastuzumab}

Trastuzumab is a monoclonal antibody that targets the HER2 receptor and is mainly used in the treatment of HER2-positive breast cancers and HER2positive cancers involving the gastroesophageal junction. However, its role in BTC is not well defined. In a retrospective analysis, Javle et al. evaluated BTC patients with HER2 genetic aberrations or protein overexpression who received HER2-directed therapy in combination with concurrent therapy of 
physician's choice. Of the eight GBC patients who received trastuzumab, $\mathrm{CR}$ was seen in one patient, while there were five patients with $\mathrm{PR}$, one with mixed response, and one with stable disease. All five cholangiocarcinoma patients had disease progression while on trastuzumab as part of its treatment [55].

\section{Lapatinib}

Lapatinib is an orally administered, dual tyrosine kinase inhibitor against EGFR and HER2. It was tested in a phase II study in patients with advanced BTC but found to have no response. Median PFS was 1.8 months and median OS was 5.2 months [56].

\section{VEGF pathway}

VEGF facilitates tumorigenesis in a variety of tumors, including BTC. Its function is not just limited to angiogenesis and vascular permeability, but also mediates signaling in tumor cells [57]. It is highly expressed in BTC, but expression varies according to the tumor type. In one study, $75 \%$ of GBC express VEGF. VEGF appears to be correlated with more advanced and metastatic stages, and its expression is associated with poor prognosis [58].

\section{Bevacizumab}

Bevacizumab is a recombinant humanized monoclonal antibody directed against VEGF. It has activity in various solid tumors in the metastatic setting, including colorectal, lung, breast, renal, and ovarian. In addition to the bevacizumab plus erlotinib trial described by Lubner et al. [43], bevacizumab's role in BTC has been investigated in phase II studies in combination with other agents. Zhu et al. enrolled 35 patients who were given bevacizumab followed by gemcitabine and oxaliplatin. Median PFS was 7.0 months. FDG-PET scans demonstrated a significant decrease in maximum standardized uptake value (SUV (max)) after only 2 cycles of treatment [59]. Iyer et al. investigated yet another regimen and recently presented data looking at gemcitabine, capecitabine, and bevacizumab in patients with advanced BTC in the first-line setting. Median PFS of 8.1 months and OS of 11.3 months were similar to standard therapy [60].

Cediranib is a potent inhibitor of the VEGF receptor tyrosine kinases and also with some activity against platelet-derived growth factor (PDGF) receptors and c-Kit [61]. Given that VEGF is overexpressed in BTC and VEGFR1 and VEGFR2 are overexpressed in adjacent endothelial cells, cediranib has also been investigated in a recent phase II study [58, 62]. Subjects were randomized to receive cisplatin and gemcitabine plus either cediranib or placebo. No statistically significant difference in median PFS was observed: 7.7 months (95 \% CI 6.3$9.3)$ in the cediranib arm vs. 7.4 months (95\% CI 5.7-8.6) in the placebo arm. There was a trend towards longer OS in the cediranib arm (14.1 months, $95 \%$ 
CI 10.2-16.0) compared to the placebo arm (11.9 months, $95 \%$ CI 9.2-13.4). However, it did appear to improve the ORR in this trial: $43 \%$ in the cediranib arm compared to $19 \%$ in the placebo arm [63•]. A phase II trial investigating cediranib in combination with modified FOLFOX6 in advanced BTC is now underway [64].

\section{Sorafenib}

Sorafenib is an oral multikinase inhibitor that inhibits cell surface tyrosine kinase receptors, including VEGF receptors and PDGF receptor- $\beta$, and blocks downstream intracellular serine/threonine kinases, such as Raf-1, wild-type, and mutant B-Raf, involved in tumor cell proliferation and angiogenesis [65]. It has been shown to be effective in hepatocellular carcinoma and renal cell carcinoma, but the same has not been demonstrated in advanced BTC. In one study, ORR was only $2 \%$, median PFS was 2.3 months, and median OS was 4.4 months [66]. Another trial involving sorafenib was terminated early given that it failed to meet the primary objective or response probability. No objective responses were observed. Median PFS was 3 months and median OS was 9 months [67].

Other trials have evaluated sorafenib in combination with standard chemotherapy. Moehler et al. investigated gemcitabine with either sorafenib or placebo in the first-line setting. However, longer median PFS and OS were actually seen in the gemcitabine plus placebo arm [68]. A randomized, double-blinded, multicenter phase II trial by Krege et al. comparing a combination of gemcitabine, cisplatin with either sorafenib or placebo found no significant difference in PFS and OS between the two arms [69]. Finally, Lee et al. compared cisplatin, gemcitabine, and sorafenib to historical data and showed no improvement in efficacy, but there was an increase in toxicity [70].

Sunitinib is another oral small molecule inhibitor that targets multiple intracellular and receptor protein kinases, including VEGF and PDGF receptors, c-Kit, and rearranged during transfection (RET). It is approved for gastrointestinal stromal tumor and renal cell carcinoma [71]. In a recent phase II study, Yi et al. investigated the role of sunitinib as a secondline treatment in advanced BTC. Only marginal efficacy was demonstrated. ORR was $8.9 \%$ and median PFS was 1.7 months [72].

Vandetanib is an oral multikinase inhibitor that targets VEGF, EGFR, as well as the RET kinase. Its role in the treatment of thyroid cancer has been well documented. In an Italian (VanGogh) study, 165 BTC patients who had not received any prior chemotherapy were randomized into three groups: vandetanib monotherapy $(\mathrm{V})$, vandetanib plus gemcitabine $(\mathrm{V} / \mathrm{G})$, and gemcitabine plus placebo (G/P). Median PFS was 105 days (95\% CI 72-155), 114 days (95\% CI 91-193), and 148 days (95\% CI 71-225), while median OS was 228 days (95 \% CI 190-364), 284 days (95 \% CI 213-359), and 307 days 


\section{MEK pathway}

(95\% CI 254-523) for V, V/G, and G/P, respectively. This study did not demonstrate any superiority of vandetanib alone or in association with gemcitabine when compared with gemcitabine alone [73•].

In addition to targeting growth factor receptors, recent research has also focused on interfering with various signaling pathways essential to the regulation of cellular processes. These include the RAS/RAF/mitogen-activated protein kinase kinase (MEK)/extracellular signal-related kinase (ERK) signaling pathway, which is activated by a diverse group of extracellular signals, such as growth factor receptors and cytokines. Activated RAS starts a phosphorylation cascade involving RAF kinase, MEK1/MEK2, and ERK1/ERK2 [74]. Phosphorylated ERKs form homodimers and translocate to the nucleus to perform important cellular functions [75]. In preclinical studies, CI-1040, a MEK inhibitor, has been investigated in a panel of human cancer cell lines and showed broad activity, particularly in cell lines harboring the BRAF mutation [76]. One study reported that BRAF mutations were identified in $22 \%$ of cholangiocarcinoma, suggesting the potential role of MEK inhibitors in the management of BTC [77].

Selumetinib is a second-generation, potent, uncompetitive inhibitor of MEK1/2. It was investigated in a phase II study for patients with advanced BTC. Median PFS was 3.7 months and OS was 9.8 months. The absence of phosphorylated ERK staining was associated with a lack of response [78]. Binimetinib, another potent, uncompetitive inhibitor of MEK1/2, was investigated in phase I trials both alone and in combination with standard chemotherapy, with encouraging activity and tolerable safety profile [79, 80]. Phase II studies exploring this drug in BTC are ongoing (NCT02151084,

NCT01828034).

\section{PIK3CA/PTEN/AKT/mTOR pathway}

The phosphatidylinositol 3-kinase (PIK3CA)/phosphatase and tensin homologue (PTEN)/AKT/mammalian target of rapamycin (mTOR) pathway is important for cell growth and survival. Abnormal activation and genetic mutations in this pathway predispose to the development of many cancers, and hence, this pathway has gained importance in recent years as a target for drug development. A study revealed that $12.5 \%$ of patients with GBC have activating mutations in PIK3CA [81]. Deregulation of this pathway has been shown to induce GBC in normal gallbladder epithelial cells [82]. Buparlisib (BKM120), a PI3K inhibitor, is being tested in various phases of clinical trials in several malignancies either as a single agent or combined with other targeted agents or conventional chemotherapy.

mTOR is upregulated in many cancer types and is currently indicated for the treatment of advanced breast cancer, neuroendocrine tumors of pancreatic origin, and renal cell carcinoma [83]. In a phase II Italian study, 39 patients with locally advanced, metastatic, or recurrent BTC progressing despite previous chemotherapy received everolimus, an inhibitor of mTOR. ORR was only $5.1 \%$, median PFS was 3.2 months, and median OS was 7.7 months [84]. In a phase II Australian study, everolimus was administered in the first-line setting in 
advanced BTC. Preliminary results were recently presented, with ORR $12 \%$, median PFS 6.0 months, and median OS 9.5 months [85]. Larger studies are necessary to clarify the efficacy of mTOR inhibitors in the treatment of BTC.

Heat shock protein (HSP90) is a chaperone protein that helps maintain structural integrity and function of specific protein targets involved in cell cycle. It has antiapoptotic activity and can target multiple pathways with promising role in many malignancies. The combination of HSP90 inhibitor and PI3K/ mTOR inhibitor was synergistic, inducing cell death in preclinical models of cholangiocarcinoma [86].

\section{Hedgehog pathway}

\section{Notch pathway}

The Hedgehog signaling pathway is involved in numerous developmental processes including cell patterning, cell fate, proliferation, survival, and differentiation during early development [87]. This pathway is dormant in most adult tissues, but its activation has been implicated in carcinogenesis. The components of the Hedgehog pathway include three secreted ligands (Sonic $\mathrm{Hh}$, Indian Hh, and Desert Hh), a negative regulatory receptor (Patched [PTCH]), a positive regulatory receptor (smoothened [SMO]), and gliomaassociated oncogene transcription factors. Nevoid basal cell carcinoma syndrome consisting of multiple basal cell carcinomas is associated with germline loss of function of mutation in PTCH. PTCH 1 and 2 mutations are also found in $30 \%$ of sporadic basal cell carcinomas [88]. Vismodegib, a small molecule that inhibits the Hedgehog pathway, is approved in basal cell carcinoma and is being studied in phase I and II trials in solid tumors [89]. Blockage of the Hedgehog pathway has been shown to decrease survival and proliferation of cancer cells in cholangiocarcinoma cell lines [90].

\section{Immunotherapy}

The Notch pathway is another attractive target in cancer therapy. It is essential for tumor angiogenesis and is needed to establish and maintain stem cell population. It has an established role in hematologic malignancies [91]. Notch signaling can be oncogenic or tumor suppressive depending on the cellular context. Mammals have four notch receptors (1-4) and five ligands. Alteration of Notch pathways in solid and liquid tumors can lead to oncogenesis [92].

A study revealed that aberrant expression of Notch receptors 1 and 3 may play a role in the cancer progression of BTC [93]. RO-4929097 (a notch signaling inhibitor) is being tested in various phase I and II trials in solid tumors (Table 2). Further research is needed to determine if this pathway can be used as a potential target in BTC.

Tumors suppress the immune system in the tumor microenvironment and also systemically. The immune system responds to cancer by reacting against tumorspecific or tumor-associated antigens (TAA). Major developments in immunological techniques have helped us discover that the immune system does 
recognize cancer antigens. Many TAA used in cancer therapeutics have been developed in recent years. Passive immunity is using monoclonal antibodies against tumor antigens or infusion of tumor-specific autologous $\mathrm{T}$ cells. Induction of active immunity by vaccination, which boosts tumor-specific antibodies and T cells, is being studied [94].

MUC1 is a large membrane glycoprotein that is identified as a tumor antigen. The MUC1 peptide vaccine has been evaluated in pancreatic and BTC in two separate trials. Yamamoto et al. evaluated eight eligible patients with metastatic disease. Seven had progressive disease and one had stable disease with a tendency for increased circulating anti-MUC1 IgG antibody after vaccination [95]. Lepisto et al. conducted a phase I/II trial using MUC1 peptideloaded dentritic cells (DC) vaccine in the adjuvant setting in BTC or pancreatic cancer. The vaccine was well tolerated with no significant toxicities. Twelve patients were enrolled and four of them were alive at 4 years [96].

Subsequently, Shimizu et al. conducted a phase I trial on 36 patients with IHCC after resection. Patients were vaccinated with autologous tumor lysate pulsed DC plus ex vivo activated T-cell transfer. Median PFS and OS were 18.3 and 31.9 months in patients receiving adjuvant immunotherapy and 7.7 and 17.4 months in the group receiving surgery alone ( $p=0.005$ and 0.022 , respectively) [97]. Further randomized controlled trials are needed to study the effects of immunotherapy in BTC.

\section{Others}

The fibroblast growth factor receptor (FGFR) pathway is involved in cellular migration, proliferation, survival, and differentiation. Various mutational profiling studies in cholangiocarcinoma have detected genetic alterations in the FGFR pathway, exclusively in IHCC, and are shown to be associated with a more indolent course $[98,99]$. FGFR2 fusions occur in $13.6 \%$ of IHCC [100]. BGJ 398, an oral FGFR inhibitor, is being evaluated in a phase II trial in patients with FGFR2 gene fusion/translocation [101]. A number of other clinical trials involving FGFR small molecule inhibitors in BTC are in progress (Table 2).

Isocitrate dehydrogenase (IDH) catalyzes the oxidative decarboxylation of isocitrate to $\alpha$-ketoglutarate ( $\alpha-\mathrm{KG}$ ). $\alpha$-KG, a substrate for multiple enzymes, is important for cellular response to oxidative stress. IDH mutation results in accumulation of D-2-hydroxyglutarate, predisposing cells to malignant transformation. These mutations are commonly found in gliomas and acute myeloid leukemias. A study reported IDH 1 and 2 mutations in IHCC ( 9 of 40, $23 \%$ ), but none in EHCC and GBC cases [102•]. The effect of IDH inhibitors is currently being evaluated in clinical trials involving solid tumors such as cholangiocarcinoma that harbor these mutations [103, 104].

Ribonucleotide reductase is an enzyme involved in DNA synthesis. 3Aminopyridine-2-carboxaldehydethiosemicarbazone (3-AP), a new, potent, small molecule inhibitor of ribonucleotide reductase was tested in combination with gemcitabine in BTC. ORR was seen in 3 of the 23 tested patients (13\%) [105].

GBC are twice more common in females and expression of estrogen receptors in GBC has been shown. Targeting estrogen receptors could have some role in the treatment, though the exact mechanism of how these receptors promote GBC is unknown [106]. 


\section{Conclusion}

BTC are rare malignancies in western countries but very prevalent in northern India and Chile. GBC, the most aggressive cancer among all, is categorized as an orphan disease. Despite recent advances in our knowledge on the pathogenesis of BTC at the molecular level, prognosis remains poor. The Cancer Genome Atlas (TCGA) helps identify genomic changes in specific tumor types and develop an integrated picture of commonalities and differences across tumor lineages [107]. BTC are currently being investigated in TCGA. Understanding the genomic alterations may help us better understand the pathogenesis and identify targetable mutations. Finally, the difficulty in the classification, molecular heterogeneity, and rarity of this disease makes clinical trials more challenging. Detection of tumors at earlier stages and improvement in surgical techniques are needed, but advancement of systemic treatment is also necessary. Targeted drugs against ErbB, VEGF, PI3K/AKT, MEK/ERK, Hedgehog, and Notch pathways are being investigated in BTC (Table 2). Enrollment of patients in clinical trials and close collaboration among international cancer organizations may lead to better outcomes in this aggressive disease.

\section{Compliance with Ethics Guidelines}

\section{Conflict of Interest}

Amartej Merla, Kenneth G. Liu, and Lakshmi Rajdev declare that they have no conflict of interest.

\section{Human and Animal Rights and Informed Consent}

This article does not contain any studies with human or animal subjects performed by any of the authors.

\section{Open Access}

This article is distributed under the terms of the Creative Commons Attribution 4.0 International License (http://creativecommons.org/licenses/by/4.0/), which permits unrestricted use, distribution, and reproduction in any medium, provided you give appropriate credit to the original author(s) and the source, provide a link to the Creative Commons license, and indicate if changes were made.

\section{References}

Papers of particular interest, published recently, have been

highlighted as:

- Of importance

• Of major importance

1. de Groen PC et al. Biliary tract cancers. N Engl J Med. 1999;341(18):1368-78.
2. Klatskin G. Adenocarcinoma of the hepatic duct at its bifurcation within the porta hepatis. An unusual tumor 
with distinctive clinical and pathological features. Am J Med. 1965;38:241-56.

3. Olnes MJ, Erlich R. A review and update on cholangiocarcinoma. Oncology. 2004;66(3):16779.

4. Lazcano-Ponce EC et al. Epidemiology and molecular pathology of gallbladder cancer. CA Cancer J Clin. 2001;51(6):349-64.

5. Castro FA et al. Biliary tract cancer incidence in the United States-demographic and temporal variations by anatomic site. Int J Cancer. 2013;133(7):1664-71.

6. Randi G et al. Epidemiology of biliary tract cancers: an update. Ann Oncol. 2009;20(1):146-59.

7. Andia KM, Gederlini GA, Ferreccio RC. Gallbladder cancer: trend and risk distribution in Chile. Rev Med Chil. 2006;134(5):565-74.

8. Altekruse SF et al. Geographic variation of intrahepatic cholangiocarcinoma, extrahepatic cholangiocarcino$\mathrm{ma}$, and hepatocellular carcinoma in the United States. PLoS One. 2015;10(3), e0120574.

9. Shaib $\mathrm{YH}$ et al. Rising incidence of intrahepatic cholangiocarcinoma in the United States: a true increase? J Hepatol. 2004;40(3):472-7.

10. Shaib Y, El-Serag HB. The epidemiology of cholangiocarcinoma. Semin Liver Dis. 2004;24(2):115-25.

11. Grainge MJ et al. The antecedents of biliary cancer: a primary care case-control study in the United Kingdom. Br J Cancer. 2009;100(1):178-80.

12. Parsi MA. Obesity and cholangiocarcinoma. World J Gastroenterol. 2013;19(4):457-62.

13. Larsson SC, Wolk A. Obesity and the risk of gallbladder cancer: a meta-analysis. Br J Cancer. 2007;96(9):145761.

14. Li M et al. Hepatitis B virus infection increases the risk of cholangiocarcinoma: a meta-analysis and systematic review. J Gastroenterol Hepatol. 2012;27(10):1561-8.

15. Welzel TM et al. Risk factors for intrahepatic and extrahepatic cholangiocarcinoma in the United States: a population-based case-control study. Clin Gastroenterol Hepatol. 2007;5(10):1221-8.

16. Jain K et al. Sequential occurrence of preneoplastic lesions and accumulation of loss of heterozygosity in patients with gallbladder stones suggest causal association with gallbladder cancer. Ann Surg. 2014;260(6):1073-80.

17. Roa I et al. Preneoplastic lesions in gallbladder cancer. J Surg Oncol. 2006;93(8):615-23.

18. Trivedi V et al. Gallbladder cancer: adenomacarcinoma or dysplasia-carcinoma sequence? Gastroenterol Hepatol (N Y). 2008;4(10):735-7.

19. Mayo SC et al. National trends in the management and survival of surgically managed gallbladder adenocarcinoma over 15 years: a population-based analysis. J Gastrointest Surg. 2010;14(10):1578-91.

20. Ribero D et al. Surgical approach for long-term survival of patients with intrahepatic cholangiocarcinoma: a multi-institutional analysis of 434 patients. Arch Surg. 2012;147(12):1107-13.
21. Farges $\mathrm{O}$ et al. Influence of surgical margins on outcome in patients with intrahepatic cholangiocarcinoma: a multicenter study by the AFC-IHCC2009 study group. Ann Surg. 2011;254(5):824-29. discussion 830 .

22. Silva MA et al. Surgery for hilar cholangiocarcinoma; a 10 year experience of a tertiary referral centre in the UK. Eur J Surg Oncol. 2005;31(5):533-9.

23. Valle J et al. Cisplatin plus gemcitabine versus gemcitabine for biliary tract cancer. N Engl J Med. 2010;362(14):1273-81.

24. Darwish Murad S et al. Efficacy of neoadjuvant chemoradiation, followed by liver transplantation, for perihilar cholangiocarcinoma at 12 US centers. Gastroenterology. 2012;143(1):88-98. e3; quiz e14.

25. Agrawal $\mathrm{S}$ et al. Outcomes of adjuvant chemoradiation and predictors of survival after extended cholecystectomy in gall bladder carcinoma: a single institution experience from an endemic region. J Gastrointest Cancer. 2015;46(1):48-53.

26. Groot Koerkamp B, Fong Y. Outcomes in biliary malignancy. J Surg Oncol. 2014;110(5):585-91.

27. Glimelius B et al. Chemotherapy improves survival and quality of life in advanced pancreatic and biliary cancer. Ann Oncol. 1996;7(6):593-600.

28. Valle JW et al. Gemcitabine alone or in combination with cisplatin in patients with advanced or metastatic cholangiocarcinomas or other biliary tract tumours: a multicentre randomised phase II study - the UK ABC01 Study. Br J Cancer. 2009;101(4):621-7.

29. Furuse J, Okusaka T, Miyazaki M, et al., A randomized study of gemcitabine/cisplatin versus single-agent gemcitabine in patients with biliary cancer. J Clin Oncol, 2009. 27(15_suppl: abstr \#4579).

30. Yang R et al. Efficacy of gemcitabine plus platinum agents for biliary tract cancers: a meta-analysis. AntiCancer Drugs. 2013;24(8):871-7.

31. Pignochino $Y$ et al. Targeting EGFR/HER2 pathways enhances the antiproliferative effect of gemcitabine in biliary tract and gallbladder carcinomas. BMC Cancer. 2010;10:631.

32. Chang YT et al. Clinicopathological and prognostic significances of EGFR, KRAS and BRAF mutations in biliary tract carcinomas in Taiwan. J Gastroenterol Hepatol. 2014;29(5):1119-25.

33. Ross JS, et al. Comprehensive genomic profiling of biliary tract cancers to reveal tumor-specific differences and genomic alterations. J Clin Oncol, 2015. 33(3_suppl: abstr \#231).

This reference presented data on comprehensive genomic profiling in BTC, including the presence/absence of genetic mutations and their relative frequencies in the subtypes of BTC.

34. Li M et al. Whole-exome and targeted gene sequencing of gallbladder carcinoma identifies recurrent mutations in the ErbB pathway. Nat Genet. 2014;46(8):872-6.

35. Kumari $\mathrm{N}$ et al. Mutation profiling in gallbladder cancer in Indian population. Indian J Pathol Microbiol. 2014;57(1):9-12. 
36. Wang SN et al. Aberrant p53 expression and the development of gallbladder carcinoma and adenoma. Kaohsiung J Med Sci. 2006;22(2):53-9.

37. Saetta AA et al. High-level microsatellite instability is not involved in gallbladder carcinogenesis. Exp Mol Pathol. 2006;80(1):67-71.

38. Normanno $\mathrm{N}$ et al. Epidermal growth factor receptor (EGFR) signaling in cancer. Gene. 2006;366(1):2-16.

39. Yoshikawa D et al. Clinicopathological and prognostic significance of EGFR, VEGF, and HER2 expression in cholangiocarcinoma. Br J Cancer. 2008;98(2):418-25.

40. Kaufman $\mathrm{M}$ et al. EGFR expression in gallbladder carcinoma in North America. Int J Med Sci. 2008;5(5):285-91.

41. Zhou YM et al. Significance of expression of epidermal growth factor (EGF) and its receptor (EGFR) in chronic cholecystitis and gallbladder carcinoma. Ai Zheng. 2003;22(3):262-5.

42. Philip PA et al. Phase II study of erlotinib in patients with advanced biliary cancer. J Clin Oncol.

2006;24(19):3069-74.

43. Lubner SJ et al. Report of a multicenter phase II trial testing a combination of biweekly bevacizumab and daily erlotinib in patients with unresectable biliary cancer: a phase II consortium study. J Clin Oncol. 2010;28(21):3491-7.

44.• Lee J et al. Gemcitabine and oxaliplatin with or without erlotinib in advanced biliary-tract cancer: a multicentre, open-label, randomised, phase 3 study. Lancet Oncol. 2012;13(2):181-8.

This is the only published phase III trial investigating the use of a targeted agent (erlotinib) in BTC, looking at erlotinib in combination with gemcitabine and oxaliplatin.

45. Gruenberger B et al. Cetuximab, gemcitabine, and oxaliplatin in patients with unresectable advanced or metastatic biliary tract cancer: a phase 2 study. Lancet Oncol. 2010;11(12):1142-8.

46. Malka D et al. Gemcitabine and oxaliplatin with or without cetuximab in advanced biliary-tract cancer (BINGO): a randomised, open-label, non-comparative phase 2 trial. Lancet Oncol. 2014;15(8):819-28.

A randomized phase II trial evaluating the role of cetuximab in combination with gemcitabine/oxaliplatin in advanced BTC.

47. Chen JS et al. A KRAS mutation status-stratified randomized phase II trial of gemcitabine and oxaliplatin alone or in combination with cetuximab in advanced biliary tract cancer. Ann Oncol. 2015;26(5):943-9.

A randomized phase II trial evaluating the role of cetuximab in combination with gemcitabine/oxaliplatin in advanced BTC.

48. Rubovszky G et al. Cetuximab, gemcitabine and capecitabine in patients with inoperable biliary tract cancer: a phase 2 study. Eur J Cancer. 2013;49(18):3806-12.

49. Hezel AF et al. Phase II study of gemcitabine, oxaliplatin in combination with panitumumab in KRAS wild-type unresectable or metastatic biliary tract and gallbladder cancer. Br J Cancer. 2014;111(3):430-6.

50. Leone F, et al. A phase II, open-label, randomized clinical trial of panitumumab plus gemcitabine and oxaliplatin (GEMOX) versus GEMOX alone as first- line treatment in advanced biliary tract cancer: the Vecti-BIL study. J Clin Oncol, 2015. 33(3_suppl: abstr \#281).

51. Jensen LH et al. Phase II marker-driven trial of panitumumab and chemotherapy in KRAS wild-type biliary tract cancer. Ann Oncol. 2012;23(9):2341-6.

52. Sohal DP et al. A phase II trial of gemcitabine, irinotecan and panitumumab in advanced cholangiocarcinoma. Ann Oncol. 2013;24(12):3061-5.

53. Menard S et al. Role of HER2/neu in tumor progression and therapy. Cell Mol Life Sci. 2004;61(23):2965-78.

54. Kiguchi $\mathrm{K}$ et al. Constitutive expression of ErbB-2 in gallbladder epithelium results in development of adenocarcinoma. Cancer Res. 2001;61(19):6971-6.

55. Javle $\mathrm{M}$ et al. HER2/neu-directed therapy for biliary tract cancer. J Hematol Oncol. 2015;8:58.

56. Ramanathan RK et al. A phase II study of lapatinib in patients with advanced biliary tree and hepatocellular cancer. Cancer Chemother Pharmacol. 2009;64(4):777-83.

57. Goel HL, Mercurio AM. VEGF targets the tumour cell. Nat Rev Cancer. 2013;13(12):871-82.

58. Quan ZW et al. Association of p53, p16, and vascular endothelial growth factor protein expressions with the prognosis and metastasis of gallbladder cancer. J Am Coll Surg. 2001;193(4):380-3.

59. Zhu AX et al. Efficacy and safety of gemcitabine, oxaliplatin, and bevacizumab in advanced biliary-tract cancers and correlation of changes in 18-

fluorodeoxyglucose PET with clinical outcome: a phase 2 study. Lancet Oncol. 2010;11(1):48-54.

60. Iyer RV, et al. Gemcitabine (G), capecitabine (C) and bevacizumab (BV) in patients with advanced biliary cancers (ABC): final results of a multicenter phase II study. J Clin Oncol, 2015. 33(15_suppl: abstr \#4078).

61. Wedge SR et al. AZD2171: a highly potent, orally bioavailable, vascular endothelial growth factor receptor-2 tyrosine kinase inhibitor for the treatment of cancer. Cancer Res. 2005;65(10):4389-400.

62. Benckert $\mathrm{C}$ et al. Transforming growth factor beta 1 stimulates vascular endothelial growth factor gene transcription in human cholangiocellular carcinoma cells. Cancer Res. 2003;63(5):1083-92.

63. Valle J, et al. ABC-03: a randomized phase II trial of cediranib (AZD2171) or placebo in combination with cisplatin/gemcitabine (CisGem) chemotherapy for patients (pts) with advanced biliary tract cancer (ABC). J Clin Oncol, 2014. 32(15_suppl: abstr \#4002).

A randomized phase II trial evaluating the role of cediranib in combination with gemcitabine/cisplatin in advanced BTC.

64. ClinicalTrials.gov (NCT01229111), Cediranib maleate and combination chemotherapy in treating patients with advanced biliary cancers.

65. Keating GM, Santoro A. Sorafenib: a review of its use in advanced hepatocellular carcinoma. Drugs. 2009;69(2):223-40.

66. Bengala $\mathrm{C}$ et al. Sorafenib in patients with advanced biliary tract carcinoma: a phase II trial. Br J Cancer. 2010;102(1):68-72. 
67. El-Khoueiry AB et al. SWOG 0514: a phase II study of sorafenib in patients with unresectable or metastatic gallbladder carcinoma and cholangiocarcinoma. Investig New Drugs. 2012;30(4):1646-51.

68. Moehler $\mathrm{M}$ et al. Gemcitabine plus sorafenib versus gemcitabine alone in advanced biliary tract cancer: a double-blind placebo-controlled multicentre phase II AIO study with biomarker and serum programme. Eur J Cancer. 2014;50(18):3125-35.

69. Krege $S$ et al. Prospective randomized double-blind multicentre phase II study comparing gemcitabine and cisplatin plus sorafenib chemotherapy with gemcitabine and cisplatin plus placebo in locally advanced and/or metastasized urothelial cancer: SUSE (AUO-AB 31/05). BJU Int. 2014;113(3):429-36.

70. Lee JK et al. A phase II study of gemcitabine and cisplatin plus sorafenib in patients with advanced biliary adenocarcinomas. Br J Cancer. 2013;109(4):915-9.

71. Kim A, Balis FM, Widemann BC. Sorafenib and sunitinib. Oncologist. 2009;14(8):800-5.

72. Yi JH et al. A phase II study of sunitinib as a second-line treatment in advanced biliary tract carcinoma: a multicentre, multinational study. Eur J Cancer. 2012;48(2):196-201.

73. Santoro A et al. A randomized, multicenter, phase II study of vandetanib monotherapy versus vandetanib in combination with gemcitabine versus gemcitabine plus placebo in subjects with advanced biliary tract cancer: the VanGogh study. Ann Oncol. 2015;26(3):542-7.

A randomized phase II trial evaluating the role of vandetanib monotherapy or in combination with gemcitabine.

74. Wellbrock C, Karasarides M, Marais R. The RAF proteins take centre stage. Nat Rev Mol Cell Biol. 2004;5(11):875-85.

75. Khokhlatchev AV et al. Phosphorylation of the MAP kinase ERK2 promotes its homodimerization and nuclear translocation. Cell. 1998;93(4):605-15.

76. Solit DB et al. BRAF mutation predicts sensitivity to MEK inhibition. Nature. 2006;439(7074):358-62.

77. Tannapfel A et al. Mutations of the BRAF gene in cholangiocarcinoma but not in hepatocellular carcinoma. Gut. 2003;52(5):706-12.

78. Bekaii-Saab T et al. Multi-institutional phase II study of selumetinib in patients with metastatic biliary cancers. J Clin Oncol. 2011;29(17):2357-63.

79. Finn RS, et al. A phase I study of MEK inhibitor MEK162 (ARRY-438162) in patients with biliary tract cancer. J Clin Oncol, 2012. 30(4_suppl: abstr \#220).

80. Lowery MA, et al. A phase I trial of binimetinib in combination with gemcitabine $(\mathrm{G})$ and cisplatin $(\mathrm{C})$ patients (pts) with untreated advanced biliary cancer (ABC). J Clin Oncol, 2015. 33(15_suppl: abstr \#e15125).

81. Deshpande $V$ et al. Mutational profiling reveals PIK3CA mutations in gallbladder carcinoma. BMC Cancer. 2011;11:60.

82. Lunardi A et al. Role of aberrant PI3K pathway activation in gallbladder tumorigenesis. Oncotarget. 2014;5(4):894-900.
83. Lebwohl D et al. Development of everolimus, a novel oral mTOR inhibitor, across a spectrum of diseases. Ann N Y Acad Sci. 2013;1291:14-32.

84. Buzzoni R et al. Activity and safety of RAD001 (everolimus) in patients affected by biliary tract cancer progressing after prior chemotherapy: a phase II ITMO study. Ann Oncol. 2014;25(8):1597-603.

85. Yeung YH, et al. Phase II study of everolimus monotherapy as first-line treatment in advanced biliary tract cancer: RADichol. J Clin Oncol, 2014. 32(15_suppl: abstr \#4101).

86. Chen $\mathrm{MH}$ et al. Antitumor activity of the combination of an HSP90 inhibitor and a PI3K/mTOR dual inhibitor against cholangiocarcinoma. Oncotarget. 2014;5(9):2372-89.

87. Varjosalo $M$, Taipale J. Hedgehog: functions and mechanisms. Genes Dev. 2008;22(18):2454-72.

88. Gailani MR et al. The role of the human homologue of Drosophila patched in sporadic basal cell carcinomas. Nat Genet. 1996;14(1):78-81.

89. ClinicalTrials.gov (NCT01546519). A study of the Hedgehog pathway inhibitor vismodegib in patients with advanced solid malignancies including hepatocellular carcinoma with varying degrees of renal or hepatic function. clinical trials.gov.

90. Jinawath A et al. Dual blockade of the Hedgehog and ERK1/2 pathways coordinately decreases proliferation and survival of cholangiocarcinoma cells. J Cancer Res Clin Oncol. 2007;133(4):271-8.

91. South AP, Cho RJ, Aster JC. The double-edged sword of Notch signaling in cancer. Semin Cell Dev Biol. 2012;23(4):458-64

92. Lobry $\mathrm{C}$ et al. Notch signaling: switching an oncogene to a tumor suppressor. Blood. 2014;123(16):2451-9.

93. Yoon HA et al. Clinicopathological significance of altered Notch signaling in extrahepatic cholangiocarcinoma and gallbladder carcinoma. World J Gastroenterol. 2011;17(35):4023-30.

94. Rabinovich GA, Gabrilovich D, Sotomayor EM. Immunosuppressive strategies that are mediated by tumor cells. Annu Rev Immunol. 2007;25:267-96.

95. Yamamoto K et al. MUC1 peptide vaccination in patients with advanced pancreas or biliary tract cancer. Anticancer Res. 2005;25(5):3575-9.

96. Lepisto AJ et al. A phase I/II study of a MUC1 peptide pulsed autologous dendritic cell vaccine as adjuvant therapy in patients with resected pancreatic and biliary tumors. Cancer Ther. 2008;6(B):955-64.

97. Shimizu K et al. Clinical utilization of postoperative dendritic cell vaccine plus activated T-cell transfer in patients with intrahepatic cholangiocarcinoma. J Hepatobiliary Pancreat Sci.

2012;19(2):171-8.

98. Churi CR et al. Mutation profiling in cholangiocarcinoma: prognostic and therapeutic implications. PLoS One. 2014;9(12), e115383.

99. Haga H, Patel T. Molecular diagnosis of intrahepatic cholangiocarcinoma. J Hepatobiliary Pancreat Sci. 2015;22(2):114-23. 
100. Arai Y et al. Fibroblast growth factor receptor 2 tyrosine kinase fusions define a unique molecular subtype of cholangiocarcinoma. Hepatology.

2014;59(4):1427-34

101. ClinicalTrials.gov (NCT02150967). A phase ii, single arm study of BGJ398 in patients with advanced cholangiocarcinoma.

102. Borger DR et al. Frequent mutation of isocitrate dehydrogenase (IDH) 1 and IDH2 in cholangiocarcinoma identified through broad-based tumor genotyping. Oncologist. 2012;17(1):72-9.

Presence of IDH mutations identifies a new target for therapy in IHCC.

103. ClinicalTrials.gov (NCT02481154). Study of orally administered AG-881 in patients with advanced solid tumors, including gliomas, with an IDH1 and/or IDH2 mutation.
104. ClinicalTrials.gov (NCT02073994). Study of orally administered AG-120 in subjects with advanced solid tumors, including glioma, with an IDH1 mutation.

105. Ocean AJ et al. Phase II trial of the ribonucleotide reductase inhibitor 3-aminopyridine-2carboxaldehydethiosemicarbazone plus gemcitabine in patients with advanced biliary tract cancer. Cancer Chemother Pharmacol. 2011;68(2):379-88.

106. Zhang LQ et al. Potential therapeutic targets for the primary gallbladder carcinoma: estrogen receptors. Asian Pac J Cancer Prev. 2013;14(4):2185-90.

107. Weinstein JN et al. The Cancer Genome Atlas PanCancer analysis project. Nat Genet. 2013;45(10):1113-20. 